\title{
BMJ Open Study protocol: an investigation of the prevalence of autism among adults admitted to acute mental health wards: a cross-sectional pilot study
}

\author{
Sam Tromans (D) , ${ }^{1,2}$ Guiqing Lily Yao, ${ }^{1}$ Reza Kiani, ${ }^{1,2}$ Regi Alexander, ${ }^{1,3}$ \\ Mohammed Al-Uzri, ${ }^{1,4}$ Traolach Brugha ${ }^{1,4}$
}

To cite: Tromans S, Yao GL, Kiani R, et al. Study protocol: an investigation of the prevalence of autism among adults admitted to acute mental health wards: a crosssectional pilot study. BMJ Open 2019;9:e033169. doi:10.1136/ bmjopen-2019-033169

- Prepublication history for this paper is available online. To view these files, please visit the journal online (http://dx.doi. org/10.1136/bmjopen-2019033169).

Received 30 July 2019 Revised 04 December 2019 Accepted 05 December 2019

D Check for updates

(c) Author(s) (or their employer(s)) 2019. Re-use permitted under CC BY. Published by BMJ.

${ }^{1}$ Department of Health Sciences, University of Leicester, Leicester, UK

${ }^{2}$ Learning Disability Psychiatry, Leicestershire Partnership NHS Trust, Leicester, UK

${ }^{3}$ Learning Disability Psychiatry, Hertfordshire Partnership

University NHS Foundation Trust, Norwich, UK

${ }^{4}$ General Adult Psychiatry, Leicestershire Partnership NHS Trust, Leicester, UK

Correspondence to

Dr Sam Tromans;

sjt56@leicester.ac.uk

\section{ABSTRACT}

Introduction Autism spectrum disorders (ASDs)

are associated with difficulties in social interaction, communication and restricted, repetitive behaviours. Much is known about their community prevalence among adults, data on adult inpatients within an acute mental health setting is lacking.

This pilot study aimed to estimate the prevalence of ASDs among adults admitted to acute mental health wards and to examine the association between ASDs and psychiatric and physical comorbidities within this group.

Methods and analysis A multiple-phase approach will be used. Phase I will involve testing of 200 patients and corresponding informants, using the autism quotient ( $A Q)$, the informant version of the Social Responsiveness Scale, second edition-Adult, the self and informant versions of the Adult Social Behaviour Questionnaire and the EuroQol5D-5L. Patients with intellectual disability (ID) will bypass Phase I.

Phase II will involve diagnostic testing of a subgroup of 40 patients with the Diagnostic Interview for Social and Communication Disorders, the Autism Diagnostic Observation Schedule version 2 and the ASD interview within the Schedules for Clinical Assessment in

Neuropsychiatry version $3.25 \pm 5$ patients will not have ID and be selected via stratified random sampling according to AQ score; $15 \pm 5$ patients will have ID. Phase II patients will be interviewed with the Physical Health Conditions and Mental IIIness Diagnoses and Treatment sections of the 2014 Adult Psychiatric Morbidity Survey.

Prevalence estimates will be based on the proportion of Phase II participants who satisfy the 10th revision of the International Statistical Classification of Diseases and Related Health Problems Diagnostic Criteria for Research (ICD-10-DCR) and the 5th edition of the Diagnostic and Statistical Manual of Mental Disorders (DSM-5) diagnostic criteria for ASD, adjusting for selection and non-response. Univariate analysis will be conducted for comorbidities to identify the level of their association with an ASD diagnosis.

Ethics and dissemination Study oversight is provided by the University of Leicester. The National Health Service Health Research Authority have provided written approval. Study results will be disseminated via conference presentations and peer-reviewed publications. Trial registration number ISRCTN27739943
Strengths and limitations of this study

- The study is currently limited to a single site, limiting its generalisability. However, applications to extend to other sites are currently in progress.

- The planned sample size is in keeping with a pilot study, although there are plans to expand to a larger trial on completion.

- The primary outcome measure (meeting the diagnostic criteria for autism spectrum disorders (ASDs)) is somewhat contingent on the diagnostic criteria used, although internationally recognised criteria have been used (the ICD-10-DCR and DSM-5).

- The study will be conducted under double-blind conditions, whereby neither patients nor health professionals conducting Phase II clinical assessments will have knowledge of the participants' corresponding Phase I questionnaire results.

- The study design allows consideration of patients with and without intellectual disabilities separately, in contrast to many other studies of its type.

\section{INTRODUCTION}

Autism spectrum disorders (ASDs) are lifelong neurodevelopmental conditions characterised by impairments in reciprocal social interaction and communication, as well as restricted, stereotyped and repetitive behaviours. ${ }^{1}$ These difficulties should manifest prior to 3 years of age, thus a detailed developmental history forms a key part of the diagnostic assessment process. Although originating in childhood, ASDs follow a persistent course throughout adult life. ${ }^{2}$ While the level of impairment associated with ASDs varies between individuals, the impact on both those with ASD and their families is always profound. ${ }^{3}$

The diagnostic criteria for ASD according to the ICD-10-DCR ${ }^{4}$ and DSM- $5{ }^{5}$ diagnostic classification systems are summarised in box 1 . It should be noted that the transition from DSM-IV to DSM-5 criteria for 
Box 1 Summaries of the ICD-10-DCR4 and DSM-55 diagnostic criteria for ASD. For the diagnostic criteria in full, please refer to the source texts.

ICD-10-DCR Criteria for Childhood Autism*

A. Presence of abnormal or impaired development before the age of three years

B. Qualitative abnormalities in reciprocal social interaction

C. Qualitative abnormalities in communication

D. Restricted, repetitive and stereotyped patterns of behaviour, interests and activities

E. The clinical picture is not attributable to the other varieties of pervasive developmental disorder; specific developmental disorder of receptive language with secondary socioemotional problems; reactive attachment disorder or disinhibited attachment disorder; mental retardation with some associated emotional or behavioural disorder; schizophrenia of unusually early onset; and Rett's syndrome.

\section{DSM-5 Criteria for Autism Spectrum Disorder}

A. Persistent deficits in social communication and social interaction across multiple contexts

B. Restricted, repetitive patterns of behaviour, interests or activities

C. Symptoms must be present in the early developmental period

D. Symptoms cause clinically significant impairment in social, occupational or other important areas of current functioning

E. These disturbances are not better explained by intellectual disability (ID)†

*ICD-10-DCR also recognises the diagnosis of 'atypical autism', whereby the features of abnormal development only manifest for the first time after 3 years of age (A) and/or the affected individual does not satisfy all three of the clinical diagnostic criteria (B-D).

TDSM- 5 recognises that ASD and ID frequently coexist, but that "to make comorbid diagnoses of autism spectrum disorder and intellectual disability, social communication should be below that expected for general developmental level."

ASD appears to reduce the number of individuals satisfying the diagnostic criteria, with only about $50 \%-75 \%$ of individuals maintaining diagnoses, a phenomenon which disproportionately affects higher functioning nonintellectually disabled (non-ID) individuals. ${ }^{6}$ However, the vast majority of what is known about ASD comes from research involving child populations, with less than $15 \%$ of UK-based ASD research being focused exclusively on adults, ${ }^{7}$ with a similar focus on children seen internationally. ${ }^{8}$

When autism was first described in the medical literature, it was considered a rare condition. ${ }^{9}$ However, there has been a significant rise in reported prevalence estimates in recent decades, which has been attributed to a myriad of factors, including broadening of diagnostic criteria, changes in study methodology, increasing knowledge of ASD among lay persons and professionals, and possibly a genuine increase in prevalence. ${ }^{10}$

ASDs represent a major global public health issue, responsible for over 111 disability adjusted life years per 100000 persons. ${ }^{11}$ Community-based prevalence rates for ASDs in adults within the general population show considerable variation across studies over time, although a recent large-scale epidemiological study estimated a prevalence rate of $1.1 \%$ (95\% CI 0.3 to 1.9$).{ }^{1112}$

A large UK-based study of 7274 participants in the 2014 Adult Psychiatric Morbidity Survey (APMS) ${ }^{13}$ coupled with a register of 290 adults with intellectual disability (ID), defined by DSM-5 as deficits in intellectual functioning (typified by an IQ score of $\leq 70$ ) coupled with deficits or impairments of adaptive functioning, with onset during the developmental period found an overall estimated ASD prevalence of $1.1 \% \quad(95 \%$ CI $0.3 \%$ to $1.9 \%) .{ }^{12}$ This is in keeping with the findings of a child study of 56946 participants by Baird et al, ${ }^{14}$ who found an overall estimated prevalence of ASDs of 116.1 per 10000 (95\% CI 90.4 to 141.8). However, while the Brugha $e^{a l^{12}}$ ASD prevalence findings for people with mild ID (1.0\%, $95 \%$ CI 0.4 to 2.2) were similar to the overall findings, those with moderate to profound ID had a markedly increased risk of ASD, with prevalence rates of $42.3 \%$ (95\% CI 31.1 to 54.3 ) and $35.2 \%$ (95\% CI 23.5 to 49.0 ) in male and female, respectively.

However, relative to the community setting, there has been very little research into the prevalence of ASDs among adults within acute mental health inpatient settings. ${ }^{15}$ Previous work appears to suggest that the prevalence of ASDs could be significantly greater in this group than that of the general population and that they may be substantially underdiagnosed in this group, with prevalence estimates varying from $1.5 \%$ to $9.9 \% .^{16-19}$

The vast majority of research on psychiatric comorbidity in patients with ASDs has been conducted in child and adolescent populations. ${ }^{20}$ Such studies have shown high rates of comorbid psychiatric conditions among individuals with ASDs, with rates ranging from $70.8 \%{ }^{21}$ to $80.9 \%,{ }^{22}$ and commonly comorbid conditions including anxiety disorders, obsessive compulsive disorders, phobias, oppositional defiant disorder and attention deficit hyperactivity disorder. ${ }^{21-25}$ Notably, a study by Brereton $e t a l^{25}$ found that children with ASDs experienced significantly higher levels of psychopathology compared with those with ID, a group which in itself is associated with an elevated risk of psychiatric disorder. ${ }^{26}$

However, it is unclear how the burden of psychiatric comorbidity seen in children with ASDs transitions into adult life. ${ }^{27}$ It is essential to understand this natural course of ASDs and its comorbidities in order to develop appropriate services to meet the needs of this patient group. ${ }^{28}$ Additionally, some psychiatric disorders, such as schizophrenia, do not typically manifest until late adolescence onwards,${ }^{29}$ so child comorbidity studies into such disorders would be of limited value.

A scoping review by Cashin et $a \hat{l}^{30}$ found a lack of research pertaining to the physical health of individuals with diagnosed ASDs, and that most studies were limited to retrospective analysis of healthcare records rather than involving any form of direct health assessment. Additionally, these studies were focused on individuals with previously recognised ASD diagnosed through routine clinical assessment, rather than having been identified through 


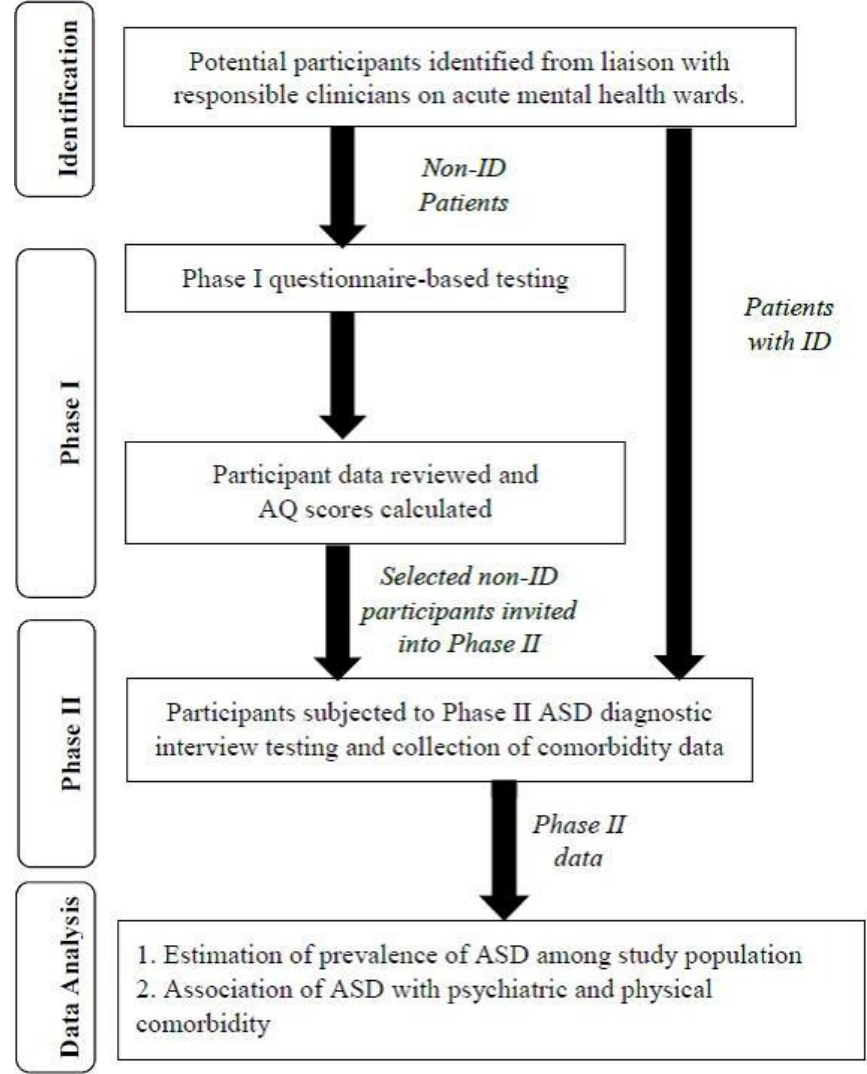

Figure 1 Flow chart summary of study design. ASDs, Autism spectrum disorders; $A Q$, autism quotient

population-based epidemiological studies. Current evidence suggests that children with ASDs are at greater risk of a range of comorbid physical illness compared with their non-ASD peers, including obesity, ${ }^{31-33}$ epilepsy, ${ }^{34}{ }^{35}$ asthma, ${ }^{35}$ sleep disorders ${ }^{36}$ and gastrointestinal disorders. ${ }^{36}$ This assertion is further supported in a recent systematic review by Muskens $e t$ al, ${ }^{37}$ who described medical disorders across a range of medical areas (including immunology, neurology and gastroenterology) as being widespread among children with ASD. However, despite the relative paucity of research in child populations, even less is known about the burden of physical comorbidity among adults with ASDs. ${ }^{38}$

The aims of this prospective pilot study are to estimate the prevalence of ASDs among adults based on acute mental health inpatient units, as well as to examine the association between ASDs and other psychiatric and physical comorbidities within this group.

\section{METHODS AND ANALYSIS \\ Study design}

The study (also known as the SPRINT study (The Prevalence of Social Communication PRoblems in Adult Psychiatric InpaTients)) will employ a multiple-phase cross-sectional design for participants without ID, and a single-phase design for participants with ID (figure 1). A multiple-phase approach to studying ASD prevalence has been used in several previous similar studies, ${ }^{12} 1939$ as the
Table 1 The probability of selection for Phase II according to total scores in the $A Q$ questionnaire. This probability framework has been validated in a previous study conducted on adult participants from a mixture of inpatient and community mental health settings. ${ }^{39}$ Participants scoring higher on these questionnaires will have a greater probability of selection

\begin{tabular}{ll|}
\hline AQ score & Probability of selection \\
\hline$\leq 19$ & 0.1 \\
$20-24$ & 0.2 \\
$25-29$ & 0.3 \\
$30-39$ & 0.6 \\
$\geq 40$ & 1.0 \\
\hline
\end{tabular}

ASD clinical interview-based diagnostic assessments are both time and resource intensive. ${ }^{40}$ Thus, a multiphase approach permits coverage of a larger study population per unit of resource. However, such an approach is heavily reliant on the accuracy of the tool used in the first phase in identifying probable cases of ASD.

In terms of the multiple-phase design for non-ID patients, transition to Phase II will be selected via stratified random sampling according to the patient's scores on the Autism Quotient (AQ $)^{41}$ questionnaire (table 1). This weighted approach enables generation of a prevalence estimate for the population as a whole (compared with if there were a single threshold AQ score for Phase II progression) and has been extensively validated, including in the 2014 APMS. $^{13}$

The rationale for a single-phase design in patients with ID is twofold. First, there is a lack of validated and relevant ASD questionnaires for this patient group. ${ }^{42}$ Second, the prevalence of ASD in this patient group is substantially higher in community studies, ${ }^{12}$ such that a single-phase design would be less resource-inefficient in identifying individuals meeting ASD diagnostic criteria within this group.

\section{Study population}

The total sample will comprise 200 patients between the ages of 18 and 65 years, with a history of having been a psychiatric inpatient on an adult acute mental health ward during the study time period (from 6 March 2019 to 31 December 2020). The inclusion and exclusion criteria are summarised in table 2. Out of these 200 patients undergoing Phase I testing, 40 will transition to Phase II of the study. The Phase II patient group will consist of $25 \pm 5$ non-ID patients, and $15 \pm 5$ patients with ID.

Study participants will be recruited from two inpatient units based in Leicestershire, UK. These units serve a population of approximately 1 million people living in both urban and rural areas within Leicester, Leicestershire and Rutland. ${ }^{43}$ Applications to extend the study to further sites are currently in progress. 
Table 2 Inclusion and exclusion criteria. Additionally, participants lacking capacity will be withdrawn from the study if they become distressed by the assessment process and/or their guardians are not in agreement with them remaining in the study

\begin{tabular}{|c|c|}
\hline Inclusion criteria & Exclusion criteria \\
\hline $\begin{array}{l}\text { Aged between } 18 \text { and } 65 \\
\text { years. }\end{array}$ & $<18$ or $>65$ years of age. \\
\hline \multirow[t]{3}{*}{$\begin{array}{l}\text { Being or having been a } \\
\text { psychiatric inpatient on an } \\
\text { adult acute mental health } \\
\text { ward during the study time } \\
\text { period. }\end{array}$} & $\begin{array}{l}\text { Having no history of being a } \\
\text { psychiatric inpatient on an } \\
\text { adult acute mental health ward } \\
\text { during the study time period. }\end{array}$ \\
\hline & Clinical diagnosis of dementia. \\
\hline & $\begin{array}{l}\text { Not understanding written } \\
\text { and/or verbal English. }\end{array}$ \\
\hline
\end{tabular}

\section{Patient and public involvement}

The study design was refined following consultation group sessions with both patients with ID and their carers, as well as patients without ID and their carers. Significant changes made to the study design were made as a result of these sessions, including reduction of the questionnaire and interview burden, as well as incorporation of a stigma measure for patients with ID, as this was considered a research priority by patients with ID and their carers.

Patients and carers will all receive a report summarising the findings of the study, unless they request not to, and an easy-read version of the report will be developed for patients with ID.

\section{Measures}

The measures to be used are summarised in table 3 . The Phase I measures, which will be used to test non-ID participants only, include the $\mathrm{AQ}{ }^{41}$ which is recommended by the National Institute for Health and Care Excellence as referral tools for adults with possible ASD who do not have ID. ${ }^{44}$ Also included in the test battery are ASD tools which are less comprehensively validated in adult populations, but appear to show promise, including the Informant version of the Social Responsiveness Scale, second edition $^{45}$ and the Self-report and Informant versions of the Adult Social Behaviour Questionnaire, ASBQ. ${ }^{46}$ Phase I also includes a basic information form for participants,

Table 3 Summary of study measures

\begin{tabular}{|c|c|}
\hline Measure & Purpose \\
\hline \multicolumn{2}{|l|}{ Phase I (non-ID participants only) } \\
\hline Autism Quotient, $\mathrm{AQ}^{41}$ & Measuring likelihood of ASD. \\
\hline \multicolumn{2}{|l|}{$\begin{array}{l}\text { Self-report and Informant versions of the Adult Social } \\
\text { Behaviour Questionnaire, } \mathrm{ASBQ}^{46}\end{array}$} \\
\hline Basic information form & $\begin{array}{l}\text { Collecting information pertaining to patient demographics, as } \\
\text { well as the } 2014 \text { Adult Psychiatric Morbidity Survey (APMS) } \\
\text { mental illness and physical health conditions checklists. }{ }^{13}\end{array}$ \\
\hline \multicolumn{2}{|l|}{ Phase II } \\
\hline $\begin{array}{l}\text { The Mental IIIness Diagnoses and Treatment section of the } \\
2014 \text { APMS }^{13}\end{array}$ & $\begin{array}{l}\text { Establishing participant's psychiatric and physical health } \\
\text { comorbidities. }\end{array}$ \\
\hline \multicolumn{2}{|l|}{ The Physical Health Conditions section of the 2014 APMS $^{13}$} \\
\hline $\begin{array}{l}\text { ASD interview subsection of version } 3 \text { of the Schedules for } \\
\text { Clinical Assessment in Neuropsychiatry, ASD-SCAN-3 }\end{array}$ & $\begin{array}{l}\text { Field testing of interview schedule } \\
\text { (for non-ID participants only). }\end{array}$ \\
\hline $\begin{array}{l}\text { Stigma Questionnaire for people with Intellectual Disability, } \\
\text { SQID }^{50}\end{array}$ & $\begin{array}{l}\text { Participants experience of stigma } \\
\text { (for ID participants only). }\end{array}$ \\
\hline Basic information form & $\begin{array}{l}\text { Collecting information pertaining to patient demographics, as } \\
\text { well as the } 2014 \text { Adult Psychiatric Morbidity Survey (APMS) } \\
\text { mental illness and physical health conditions checklists }{ }^{13} \text { (for } \\
\text { ID participants only, as this form is completed in phase I for } \\
\text { non-ID participants). }\end{array}$ \\
\hline
\end{tabular}

ID, intellectual disability. 
Table 4 Summary of secondary outcome parameters

\begin{tabular}{|c|c|c|}
\hline Outcome category & Corresponding parameters & $\begin{array}{l}\text { Study phase in which data is } \\
\text { collected }\end{array}$ \\
\hline $\begin{array}{l}\text { Basic demographic } \\
\text { information }\end{array}$ & $\begin{array}{l}\text { Date of birth } \\
\text { Sex } \\
\text { Postcode } \\
\text { Ethnic group } \\
\text { Employment status } \\
\text { Relationship status }\end{array}$ & $\begin{array}{l}\text { Phase I (non-ID participants) } \\
\text { Phase II (ID participants) }\end{array}$ \\
\hline \multirow[t]{2}{*}{ Mental health history } & $\begin{array}{l}\text { Date of most recent psychiatric hospital admission } \\
\text { Discharge date of most recent psychiatric hospital } \\
\text { admission (where applicable) } \\
\text { Total number of inpatient psychiatric admissions } \\
2014 \text { Adult Psychiatric Morbidity Survey (APMS) mental } \\
\text { illness checklist }^{13}\end{array}$ & $\begin{array}{l}\text { Phase I (non-ID participants) } \\
\text { Phase II (ID participants) }\end{array}$ \\
\hline & $\begin{array}{l}\text { The Mental Illness Diagnoses and Treatment section of the } \\
2014 \text { APMS }^{13}\end{array}$ & - Phase II \\
\hline \multirow[t]{2}{*}{ Physical health history } & - 2014 APMS physical health conditions checklist ${ }^{13}$ & $\begin{array}{l}\text { Phase I (non-ID participants) } \\
\text { - Phase } 2 \text { (ID participants) }\end{array}$ \\
\hline & $\begin{array}{l}\text { The Physical Health Conditions section of the } 2014 \\
\text { APMS }^{13}\end{array}$ & - Phase II \\
\hline General health & - EuroQol-5D-5L ${ }^{47}$ data for participants and informants & - Phase I \\
\hline $\begin{array}{l}\text { Stigma experience (ID } \\
\text { participants only) }\end{array}$ & $\begin{array}{l}\text { Stigma Questionnaire for people with Intellectual Disability, } \\
\text { SQID }^{50}\end{array}$ & - Phase II (ID participants) \\
\hline
\end{tabular}

ID, intellectual disability.

including demographic details, encompassing basic demographic information and information pertaining to mental and physical health, including the 2014 APMS mental illness and physical health conditions checklists, thus enabling measurement harmonisation and wider comparison. ${ }^{13}$ Additionally, both participants and informants will complete the EuroQol-5D-5L, ${ }^{47}$ a self-report instrument that measures general health.

The Phase II measures include the DISCO ${ }^{48}$ (although the interview will be limited to the diagnostic algorithm, psychiatric conditions and forensic problems items) and ADOS-2, ${ }^{49}$ both of which are tools with established validity for the diagnosis of ASDs. Also, psychiatric and physical comorbidities will be assessed using the relevant sections of the 2014 APMS interview. ${ }^{13}$ Other tests include the field testing of the ASD interview of version 3 of the Schedules for Clinical Assessment in Neuropsychiatry, as well as the Stigma Questionnaire for people with Intellectual Disability. ${ }^{50}$

\section{Outcome parameters}

The primary outcome parameter for this pilot study is the presence or absence of meeting ICD-10-DCR and DSM-5 diagnostic criteria for ASDs, respectively. Secondary outcome parameters, including the study phase in which the corresponding parameters are collected, are summarised in table 4 .

\section{Data analysis plan}

The primary outcome parameter will be based on patients who transition to Phase II of the study. The ASD prevalence estimate will be calculated based on the proportion of Phase II participants who meet the ICD-10-DCR and DSM-5 diagnostic criteria for ASD on clinical assessment, with adjustment for selection and non-response, as well as the different study designs for the ID and non-ID patient subgroups.

For the feasibility study, the analyses will be descriptive. For continuous variables, values and SD will be reported; for categorical variables, percentages and SD will be reported. We will use multiple imputation to account for any missing data. For the full-powered study, the generalised linear model will be used to estimate the difference between groups, as well as corresponding OR, 95\% CI and $p$ values.

The analyses will be primarily conducted in SPSS (version 26), but for more advanced statistical analyses (such as use of the generalised linear model) and graphical representation, we will use $\mathrm{R}$. 


\section{Sample size justification}

The sample size was calculated with direct statistician support, as well as the use of a statistical textbook and related software.$^{51}$ For a significance level of 0.05 and power of $85 \%$, assuming estimates of $1 \%$ community ASD prevalence and $5 \%$ inpatient ASD prevalence, ${ }^{15}$ a sample size of 374 patients is required. Ideally, we would be subjecting a minimum of 374 patients to Phase II testing. This is because only participants completing Phase II interview assessments will yield data pertaining to the primary outcome variable (ie, the presence or absence of satisfying diagnostic criteria for ASD).

However, in the first instance, around 40 patients (from the minimum of 200 subjected to Phase I testing) will be selected for Phase II testing (via stratified random sampling for non-ID patients, whereas ID patients will progress automatically to Phase II). The Phase I sample size of 200 patients takes into account the probability sampling method for Phase II selection, in addition to allowing for losses to follow-up for Phase I participants invited for Phase II testing. Our results will assume that the data for these 40 patients are representative of the larger group subjected to Phase I testing. Owing to the sample size, the SPRINT study shou

ld be considered a pilot/feasibility study, the intention of which being to establish and further refine the technical, administrative and logistical aspects of the study, with a view towards sample size expansion on its completion. The sample size represents an appropriate sample size for a feasibility study of this type. ${ }^{5253}$

\section{Ethics and dissemination}

The written approval of the National Health Service Health Research Authority has been obtained, as well as the local ethics committee of the study centre. Written informed consent is and will be obtained from every participant. Significant modifications to the study protocol will be communicated to relevant members of the research team. Any results from this study will be published in peer-reviewed journals and conference proceedings. Additionally, the study's findings will be disseminated to all participants, including easy-read versions of the findings for those with IDs.

\section{Twitter Sam Tromans @SamuelJTromans}

Acknowledgements The authors would like to acknowledge the following individuals for their contributions to the study: Nicola Spiers, Maria Viskaduraki and Zoe Morgan for statistical support. Elizabeta Mukaetova-Ladinska and Verity Chester for assistance with the systematic reviews underpinning the study. Dave Clarke and Henry Simkins for assistance with study design. Natalie Marking, Hannah Harrison, Tom Pringle, Deborah Glancy, Joanna McGarr, Rebekah Pole, Kris Roberts and Sam Pollen for support with study recruitment. Debra Bugler, Janice Holmes, Joy Fellows and Robin Oxley-Boyle for administrative support. Joanne Prosser and Naishali Chandarana for advice and support in developing easy-read materials. Freya Tyrer and Lesley Thoms for assistance with Patient and Public Involvement Sessions. The members of the Peoples Forum (Leicester, United Kingdom) and patients and carers at Leicestershire Partnership NHS Trust for providing valued input in the Patient and Public Involvement Sessions.

Contributors ST, TB, GLY, RK, RA and MA-U were involved in drafting the study protocol. TB and ST conceived of the idea for this study. ST is the principal investigator for the study, under the supervision of TB. ST led in writing the manuscript, TB and GLY contributed revisions and all authors approved the final manuscript.

Funding This study was supported by funding awarded by an NIHR Senior Investigator, as well as receiving funding and resource support from the NIHR, as a result of the study being adopted onto the NIHR Clinical Research Network Portfolio of studies. GLY's research is partly funded by an MRC grant.

Competing interests TB is directly involved with the development of the autism spectrum disorder (ASD) interview questions for the Schedules for Assessment in Neuropsychiatry version 3, which have not yet been formally published; this study is involved in testing the clinical utility of these questions. Additionally, TB and RK were authors of the 2014 APMS, from which two questionnaires are being used. Neither TB nor RK will be receiving any monetary payment as a result of the inclusion of the ASD interview questions or the 2014 APMS questionnaires within this study.

Patient consent for publication Not required.

Provenance and peer review Not commissioned; externally peer reviewed.

Open access This is an open access article distributed in accordance with the Creative Commons Attribution 4.0 Unported (CC BY 4.0) license, which permits others to copy, redistribute, remix, transform and build upon this work for any purpose, provided the original work is properly cited, a link to the licence is given, and indication of whether changes were made. See: https://creativecommons.org/ licenses/by/4.0/

ORCID iD

Sam Tromans http://orcid.org/0000-0002-0783-285X

\section{REFERENCES}

1 World Health Organization. The ICD-10 classification of mental and behavioural disorders: clinical descriptions and diagnostic guidelines. Geneva: World Health Organization, 1992.

2 Howlin P. Outcome in adult life for more able individuals with autism or Asperger syndrome. Autism 2000;4:63-83.

3 Newschaffer CJ, Croen LA, Daniels J, et al. The epidemiology of autism spectrum disorders. Annu Rev Public Health 2007;28:235-58.

4 World Health Organization. The ICD-10 classification of mental and behavioural disorders: diagnostic criteria for research. Geneva: World Health Organization, 1993.

5 American Psychiatric Association. Diagnostic and statistical manual of mental disorders: DSM-5. Arlington: American Psychiatric Publishing, 2013

6 Smith IC, Reichow B, Volkmar FR. The effects of DSM- 5 criteria on number of individuals diagnosed with autism spectrum disorder: a systematic review. J Autism Dev Disord 2015;45:2541-52.

7 MacLeod A, Guldberg K, Kossyvaki L, et al. Mind the gap: what is missing in the autism research agenda? Available: https://www. birmingham.ac.uk/research/perspective/autism-research-experts. aspx [Accessed $12 \mathrm{Jul}$ 2019].

8 Lever AG, Geurts HM. Psychiatric co-occurring symptoms and disorders in young, middle-aged, and older adults with autism spectrum disorder. J Autism Dev Disord 2016;46:1916-30.

9 Kanner L, autism Einfantile. Early infantile autism. J Pediatr 1944;25:211-7.

10 Wing L, Potter D. The epidemiology of autistic spectrum disorders: is the prevalence rising? Ment Retard Dev Disabil Res Rev 2002;8:151-61.

11 Baxter AJ, Brugha TS, Erskine HE, et al. The epidemiology and global burden of autism spectrum disorders. Psychol Med 2015;45:601-13.

12 Brugha TS, Spiers N, Bankart J, et al. Epidemiology of autism in adults across age groups and ability levels. $\mathrm{Br} J$ Psychiatry 2016;209:498-503.

13 McManus S, Bebbington P, Jenkins R, et al. Mental health and wellbeing in England: adult psychiatric morbidity survey 2014. Leeds: NHS Digital, 2016.

14 Baird G, Simonoff E, Pickles A, et al. Prevalence of disorders of the autism spectrum in a population cohort of children in South Thames: the special needs and autism project (SNAP). The Lancet 2006;368:210-5.

15 Tromans S, Chester V, Kiani R, et al. The prevalence of autism spectrum disorders in adult psychiatric inpatients: a systematic review. CPEMH 2018;14:177-87.

16 Mandell DS, Lawer LJ, Branch K, et al. Prevalence and correlates of autism in a state psychiatric hospital. Autism 2012;16:557-67. 
17 Shah A, Holmes N, Wing L. Prevalence of autism and related conditions in adults in a mental handicap Hospital. Appl Res Ment Retard 1982;3:303-17.

18 Scragg P, Shah A. Prevalence of Asperger's syndrome in a secure Hospital. Br J Psychiatry 1994;165:679-82.

19 Hare DJ, Gould J, Mills R, et al. A preliminary study of individuals with autistic spectrum disorders in three special hospitals in England. London: National Autistic Society, 1999.

20 Ghaziuddin M, Zafar S. Psychiatric comorbidity of adults with autism spectrum disorders. Clin Neuropsychiatry 2008;5:9-12.

21 Simonoff E, Pickles A, Charman T, et al. Psychiatric disorders in children with autism spectrum disorders: prevalence, comorbidity, and associated factors in a population-derived sample. J Am Acad Child Adolesc Psychiatry 2008;47:921-9.

22 de Bruin El, Ferdinand RF, Meester S, et al. High rates of psychiatric co-morbidity in PDD-NOS. J Autism Dev Disord 2007;37:877-86.

23 Mattila M-L, Hurtig T, Haapsamo H, et al. Comorbid psychiatric disorders associated with Asperger Syndrome/High-functioning autism: a community- and clinic-based study. J Autism Dev Disord 2010;40:1080-93.

24 Leyfer OT, Folstein SE, Bacalman S, et al. Comorbid psychiatric disorders in children with autism: interview development and rates of disorders. J Autism Dev Disord 2006;36:849-61.

25 Brereton AV, Tonge BJ, Einfeld SL. Psychopathology in children and adolescents with autism compared to young people with intellectual disability. J Autism Dev Disord 2006;36:863-70.

26 Cooper S-A, Smiley E, Morrison J, et al. Mental ill-health in adults with intellectual disabilities: prevalence and associated factors. $\mathrm{Br} \mathrm{J}$ Psychiatry 2007;190:27-35.

27 Joshi G, Wozniak J, Petty C, et al. Psychiatric comorbidity and functioning in a clinically referred population of adults with autism spectrum disorders: a comparative study. J Autism Dev Disord 2013;43:1314-25.

28 Howlin P, Goode S, Hutton J, et al. Adult outcome for children with autism. J Child Psychol Psychiatry 2004;45:212-29.

29 Cascio MT, Cella M, Preti A, et al. Gender and duration of untreated psychosis: a systematic review and meta-analysis. Early Interv Psychiatry 2012;6:115-27.

30 Cashin A, Buckley T, Trollor JN, et al. A scoping review of what is known of the physical health of adults with autism spectrum disorder. $\mathrm{J}$ Intellect Disabil 2018;22:96-108.

31 Eaves LC, Ho HH. Young adult outcome of autism spectrum disorders. J Autism Dev Disord 2008;38:739-47.

$32 \mathrm{Ho} \mathrm{HH}$, Eaves LC, Peabody D. Nutrient intake and obesity in children with autism. Focus Autism Other Dev Disabl 1997;12:187-92.

33 Granich J, Lin A, Hunt A, et al. Obesity and associated factors in youth with an autism spectrum disorder. Autism 2016;20:916-26.

34 Levy SE, Giarelli E, Lee L-C, et al. Autism spectrum disorder and cooccurring developmental, psychiatric, and medical conditions among children in multiple populations of the United States. J Dev Behav Pediatr 2010;31:267-75.

35 Schieve LA, Gonzalez V, Boulet SL, et al. Concurrent medical conditions and health care use and needs among children with learning and behavioral developmental disabilities, National health interview survey, 2006-2010. Res Dev Disabil 2012;33:467-76.
36 Bauman ML. Medical comorbidities in autism: challenges to diagnosis and treatment. Neurotherapeutics 2010;7:320-7.

37 Muskens JB, Velders FP, Staal WG. Medical comorbidities in children and adolescents with autism spectrum disorders and attention deficit hyperactivity disorders: a systematic review. Eur Child Adolesc Psychiatry 2017;26:1093-103.

38 Croen LA, Zerbo O, Qian Y, et al. The health status of adults on the autism spectrum. Autism 2015;19:814-23.

39 Brugha T, Tyrer F, Leaver A, et al. Testing adults by questionnaire for social and communication disorders, including autism spectrum disorders, in an adult mental health service population.. Int $J$ Methods Psychiatr Res.

40 Ferriter M, Hare D, Bendall P, et al. Brief report: assessment of a screening tool for autistic spectrum disorders in adult population. $J$ Autism Dev Disord 2001;31:351-3.

41 Baron-Cohen S, Wheelwright S, Skinner R, et al. The autismspectrum quotient (AQ): evidence from Asperger syndrome/ high-functioning autism, males and females, scientists and mathematicians. J Autism Dev Disord 2001;31:5-17.

42 National Institute for Health and Care Excellence. Autism spectrum disorder in adults: diagnosis and management (CG142). Available: https://www.nice.org.uk/guidance/cg142/resources/autismspectrum-disorder-in-adults-diagnosis-and-management-pdf35109567475909 [Accessed 12 Jul 2019].

43 Leicestershire Partnership NHS Trust. Basic facts and figures. Available: http://www.leicspart.nhs.uk/_AboutusWhoWeAreandWhatWeDo-FactsandFigures.aspx [Accessed $12 \mathrm{Ju}$ 2019].

44 National Institute for Health and Care Excellence. Autism spectrum disorder in adults: Diagnosis and management. NICE guideline [CG142], 2012.

45 Constantino JN, Gruber CP. Social responsiveness scale (SRS). California: Western Psychological Services, 2012.

46 Horwitz EH, Schoevers RA, Ketelaars CEJ, et al. Clinical assessment of ASD in adults using self- and other-report: psychometric properties and validity of the adult social behavior questionnaire (ASBQ). Res Autism Spectr Disord 2016;24:17-28.

47 Herdman M, Gudex C, Lloyd A, et al. Development and preliminary testing of the new five-level version of EQ-5D (EQ-5D-5L). Qual Life Res 2011;20:1727-36.

48 Wing L, Leekam SR, Libby SJ, et al. The diagnostic interview for social and communication disorders: background, interrater reliability and clinical use. J Child Psychol Psychiatry 2002;43:307-25.

49 Lord C, Rutter M, DiLavore P, et al. Autism diagnostic observation schedule-2nd edition (ADOS-2). California: Western Psychological Corporation, 2012

50 Ali A, Strydom A, Hassiotis A, et al. A measure of perceived stigma in people with intellectual disability. Br J Psychiatry 2008;193:410-5.

51 Machin D, Campbell MJ, Tan S, et al. Sample size tables for clinical studies. New Jersey: Wiley-Blackwell, 2011.

52 Johanson GA, Brooks GP. Initial scale development: sample size for pilot studies. Educ Psychol Meas 2010;70:394-400.

53 Hertzog MA. Considerations in determining sample size for pilot studies. Res Nurs Health 2008;31:180-91. 